\title{
Tailored Nanocomposites of Polypropylene with Layered Silicates
}

\author{
Liang Xu, ${ }^{\dagger}$ Hiroyoshi Nakajima, ${ }^{*, \S}$ Evangelos Manias, ${ }^{*}, *, l l$ and \\ Ramanan Krishnamoorti* ${ }^{* \dagger, \perp}$
}

\author{
Department of Chemical and Biomolecular Engineering, University of Houston, Houston, Texas 77204, \\ Department of Materials Science and Engineering, The Pennsylvania State University, University Park, \\ Pennsylvania 16802, Polymer Nanostructures Laboratory-CSPS, A Penn State Center of Excellence, \\ The Pennsylvania State University, University Park, Pennsylvania 16802, and Department of \\ Chemistry, University of Houston, Houston, Texas 77204
}

Received February 9, 2009; Revised Manuscript Received February 24, 2009

\begin{abstract}
The melt rheological properties of layered silicate nanocomposites with maleic anhydride (MA) functionalized polypropylene are contrasted to those based on ammonium-terminated polypropylene. While the MA functionalized PP based nanocomposites exhibit solid-like linear viscoelastic behavior, consistent with the formation of a long-lived percolated nanoparticle network, the single-end ammonium functionalized PP based nanocomposites demonstrated liquid-like behavior at comparable montmorillonite concentrations. The differences in the linear viscoelasticity are attributed to the presence of bridging interactions in MA functionalized nanocomposites. Further, the transient shear stress of the MA functionalized nanocomposites in start-up of steady shear is a function of the shear strain alone, and the steady shear response is consistent with that of non-Brownian systems. The weak dependence of the steady first normal stress difference on the steady shear stress suggests that the polymer chain mediated silicate network contributes to such unique flow behavior.
\end{abstract}

\section{Introduction}

The stable and controlled dispersion of nanometer-thick layered silicates in polymer matrices, especially polyolefin matrices, remains a significant challenge in the technological applications of such materials. ${ }^{1}$ While thermodynamic considerations for preparing such nanocomposites suggest several successful strategies for the functionalization of the polyolefins and the surface modification of the silicate sheets, the realization of stable and processing-independent nanocomposite preparation with reproducible thermal, mechanical and rheological/viscoelastic properties remains an outstanding issue. ${ }^{2,3}$ Development of well-defined property suites for polyolefin based nanocomposites therefore remains a technological goal and an area of active research.

In this work, we report on the melt-state rheological properties in the context of the layered-silicate dispersion in polypropylene (PP)/montmorillonite nanocomposites, and in connection with the associated changes in crystallization and mechanical properties. Two series of model, master-batch formed, polypropylene-based nanocomposites are examined in detail, with the emphasis on correlating differences in viscoelastic and thermomechanical properties to the type of interactions between the polymer and the silicate sheets. Specifically, we compare the melt-rheological properties of nanocomposites made through montmorillonite masterbatches by single end-functionalized (ammonium terminated) polypropylene against those of maleic-anhydride functionalized PP (PP-g-MA). Previous studies have indicated that the melt-state linear rheology in polymer nanocomposites is dominated by the mesoscale dispersion of the nanoparticles

* Address correspondence to R.K. (e-mail: ramanan@uh.edu) or E.M. (e-mail: manias@psu.edu).

${ }^{\dagger}$ Department of Chemical and Biomolecular Engineering, University of Houston.

$¥$ Department of Materials Science and Engineering, The Pennsylvania State University.

${ }^{\S}$ Permanent address: Sumitomo Chemical Co. Ltd., Sodegaura, Chiba, Japan.

"Polymer Nanostructures Laboratory-CSPS, A Penn State Center of Excellence, The Pennsylvania State University.

${ }^{\perp}$ Department of Chemistry, University of Houston. and the strength of the interactions between the nanoparticles and the polymer matrix. ${ }^{4-8}$ For the case of the single endfunctionalized polymers, bridging between nanoparticles via long-lived physisorbed configurations is not possible for weakly interacting polymers, such as polyolefins, and at best entanglement induced bridging interactions might occur. ${ }^{4,9,10}$

The PP- $g$-MA based nanocomposites differ qualitatively from nanocomposites based on polymers that exhibit strong interactions with the nanoparticles, such as end-tethered polyamide6/montmorillonite nanocomposites ${ }^{4,9-12}$ and poly(vinyl alcohol)/ montmorillonite nanocomposites $;^{13}$ in these latter hybrids, each monomer repeat-unit along the polymer has strong attractive interactions with the nanoparticles. For MA-treated PP, each PP- $g$-MA chain can have at most two terminal MA groups (i.e., MA are located at one or both chain ends, with the PP-backbone itself having no strong physisorbing sites for the nanoparticles); thus, PP-g-MA can adopt bridging conformations between filler particles and possibly stabilize the entanglement induced bridging interactions. ${ }^{3,5,7,14}$ These PP- $g$-MA stabilized filler networks are similar in nature to those of hydrophilic laponite kinetically trapped in hydrophobic domains of polyurethane elastomers, ${ }^{15}$ wherein despite the strong overall unfavorable polymer/nanoparticle interactions, solvent-assisted trapping of laponite particles in an elastomeric material resulted in substantial increases in modulus and viscosity. These changes in the mechanical and viscoelastic properties for those polyurethane nanocomposites were attributed largely to the formation of jammed nanoparticle networks presumably stabilized by polymer chains that hydrogen-bond to the laponite.

Further, by diluting (melt blending) the PP-g-MA based master-batch with unfunctionalized PP of selected varied molecular weights, we were able to gain an understanding regarding the influence of molecular weight difference (between the master-batch polymer and the matrix) on controlling the crystallization, and thus the mechanical properties, as well as the mesoscale filler dispersion, and thus the rheology, of such nanocomposite systems. 
Table 1. Characteristics of the Polymers and the Nanocomposites Used in This Study

\begin{tabular}{|c|c|c|c|c|}
\hline polymer & $M_{\mathrm{w}}(\mathrm{kDa})$ & \multicolumn{2}{|c|}{ melt index (g/10min) } & $T_{\mathrm{m}}\left({ }^{\circ} \mathrm{C}\right)$ \\
\hline HPP & 243 & \multicolumn{2}{|r|}{13} & 163.9 \\
\hline MPP & 162 & \multicolumn{2}{|r|}{55} & 159.0 \\
\hline LPP & 139 & \multicolumn{2}{|c|}{120} & 162.2 \\
\hline FPP & 151 & \multicolumn{2}{|r|}{53} & 159.1 \\
\hline PP- $t-\mathrm{NH}_{3}{ }^{+}$ & 135.5 & & & 158.2 \\
\hline & \multicolumn{3}{|c|}{ Composition (wt \%) } & \multirow[b]{2}{*}{$d_{001}(\mathrm{~nm})$} \\
\hline series 1 & PP & F-PP & $2 \mathrm{C} 18 \mathrm{M}$ & \\
\hline $2 \mathrm{C} 18 \mathrm{M}$ & 0 & 0 & 100 & 2.45 \\
\hline FPP M/B ${ }^{a}$ & 0 & 85 & 15 & 2.71 \\
\hline $\mathrm{HNC}$ & 67 & 28 & 5 & 2.95 \\
\hline HBN & 70 & 30 & 0 & \\
\hline MNC & 67 & 28 & 5 & 3.01 \\
\hline MBN & 70 & 30 & 0 & \\
\hline $\mathrm{LNC}$ & 67 & 28 & 5 & 3.04 \\
\hline \multirow[t]{2}{*}{$\mathrm{LBN}$} & 70 & 30 & 0 & \\
\hline & \multicolumn{3}{|c|}{ Composition (wt \%) } & \\
\hline series 2 & $\overline{\mathrm{PP}}$ & PP- $t-\mathrm{NH}_{3}{ }^{+}$ & $\overline{\mathrm{Na}^{+} \mathrm{M}}$ & $d_{001}(\mathrm{~nm})$ \\
\hline $\mathrm{Na}^{+} \mathrm{M}$ & 0 & 0 & 100 & 1.23 \\
\hline $\mathrm{HN} 188 \mathrm{M} / \mathrm{B}^{b}$ & 0 & 90 & 10 & \\
\hline HN191 & 50 & 45 & 5 & \\
\hline HN192 & 50 & 45 & 5 & \\
\hline
\end{tabular}

${ }^{a} \mathrm{FPP} \mathrm{M} / \mathrm{B}$ is the $\mathrm{FPP} / 2 \mathrm{C} 18 \mathrm{M}$ concentrate used to prepare all systems of series $1 .{ }^{b} \mathrm{HN} 188$ is the PP- $t-\mathrm{NH}_{3}{ }^{+} / \mathrm{Na}^{+} \mathrm{M}$ concentrate used to prepare all systems of series 2 .

\section{Experimental Section}

2.1. Materials and Preparation of PP/Montmorillonite Nanocomposites. Two series of polypropylene/montmorillonite nanocomposites are explored in this study, as model systems for PP/layered-silicate nanocomposites made by the dilution of concentrates ("master-batches") by neat-unfunctionalized- polypropylene: ${ }^{3}$

The first series of nanocomposites (series 1) is based on a "concentrate" of $15 \mathrm{wt} \%$ dimethyl ditallow ammonium modified montmorillonite $(2 \mathrm{C} 18 \mathrm{M}$, a commercially available organo-montmorillonite: I44PA, CEC $\cong 1.0$ mequiv/g, Nanocor, IL) dispersed in a maleic anhydride (MA) functionalized PP (F-PP, PP- $g$-MA with $M_{\mathrm{w}}=151 \mathrm{~K}$, melt index $=53 \mathrm{~g} / 10 \mathrm{~min}$, and $\phi_{\mathrm{MA}} \approx 0.1 \mathrm{wt}$ $\%$ ); corresponding to $c a .10 \mathrm{wt} \%$ montmorillonite (inorganic) loading in the concentrate. In order to explore the effects of the relative $M_{\mathrm{w}}$ of the PP (matrix) with respect to the $M_{\mathrm{w}}$ of the MAfunctionalized-PP (in the concentrate), this "master-batch" was diluted in three isotactic PP matrices with high $(\mathrm{H})$, medium $(\mathrm{M})$, and low (L) molecular weights (denoted as HPP, MPP, LPP, with $M_{\mathrm{w}}=243 \mathrm{~K}, 162 \mathrm{~K}, 139 \mathrm{~K}$, melt index $=13,55,120 \mathrm{~g} / 10 \mathrm{~min}$, and $T_{\mathrm{m}}=163.9,159.0$, and $162.2{ }^{\circ} \mathrm{C}$, respectively). All three systems were mixed at a $2: 1$ ratio of neat PP to concentrate, resulting in nanocomposites with $67 / 28 / 5$ weight ratios of PP/F$\mathrm{PP} /$ organo-montmorillonite (denoted as HNC, MNC, LNC, to reflect the molecular weight of the PP matrix: $\mathrm{H}, \mathrm{M}$, or L, respectively). The melt-processing of the concentrates (master-batches) and their dilution (let-down) was carried out in a twin-head kneader (Brabender Plasticoder) at $200{ }^{\circ} \mathrm{C}$ and $80 \mathrm{rpm}$; the residence times were 15 (master-batch) and 20 min (dilution, let-down), respectively. In order to delineate the role of the layered silicates, the respective unfilled 30/70 F-PP/PP blends were also made and studied: specifically, $30 \mathrm{wt} \%$ F-PP (as above) was mixed in the twin-head kneader under the same conditions as the nanocomposites with 70 wt $\%$ of high, medium, and lower molecular weight isotactic-PP (these blends are denoted as HBN, MBN, LBN respectively, cf. Table 1).

The second series of nanocomposites (series 2) is based on a 90:10 by weight "concentrate" of end-functionalized PP and $\mathrm{Na}^{+}$ montmorillonite $\left(\mathrm{Na}^{+} \mathrm{M}\right)$. This system was made by combining PP$t-\mathrm{NH}_{3}{ }^{+} \mathrm{Cl}^{-}$(or PP-NH${ }^{+}, M_{\mathrm{w}}=135.5 \mathrm{~K}, d=2.3, T_{\mathrm{m}}=158.2^{\circ} \mathrm{C}$, synthesized as before ${ }^{16,17}$ and summarized in the Supporting Information and shown schematically in Schematic S-1) with 10 wt $\%$ of $\mathrm{Na}^{+}$montmorillonite (CEC $\cong 1.4$ mequiv/g, Nanocor, IL), mixed and ground together in a mortar and pestle at ambient temperature, and subsequently heated at $180{ }^{\circ} \mathrm{C}$ for $2 \mathrm{~h}$ under $\mathrm{N}_{2}$ (HN188); processes, materials, and resulting structures were as described before. ${ }^{17,18}$ Nanocomposites based on this "concentrate" were prepared by melt-intercalation as before, after this concentrate was mixed and ground together at 1:1 ratio with two commercial isotactic PPs: one with $M_{\mathrm{w}}=243 \mathrm{~K}, T_{\mathrm{m}}=163.9^{\circ} \mathrm{C}$ (HN191) and the second with $M_{\mathrm{w}}=12 \mathrm{~K}, d=2.4, T_{\mathrm{m}}=157^{\circ} \mathrm{C}$ (HN192). These mixtures were subsequently annealed at $190{ }^{\circ} \mathrm{C}$ for $2 \mathrm{~h}$ under $\mathrm{N}_{2}$, resulting in both cases in nanocomposites with 50/45/5 weight ratios of PP/PP- $t-\mathrm{NH}_{3}{ }^{+} / \mathrm{mmt}$. The characteristics of all the samples are listed in Table 1.

2.2. Structural and Rheological Characterization Methods. $\mathrm{X}$-ray diffraction (XRD) measurements were performed in a Rigaku Geigerflex powder diffractometer with a Dmax-B controller in a scanning $\theta-\theta$ geometry, with a copper source $(\mathrm{K} \alpha, \lambda=0.154 \mathrm{~nm})$ at $50 \mathrm{kV}$ and $20 \mathrm{~mA}$. All XRD specimens were compression molded. Bright field transmission electron microscopy (TEM) images were obtained with a JEOL 1200 EXII microscope operated at an accelerating voltage of $80 \mathrm{kV}$, and equipped with a Tietz F224 digital camera. Ultrathin sections $(70-100 \mathrm{~nm})$ of the nanocomposites were obtained with a microtome (Leica Ultracut UCT) equipped with a diamond knife. The sections were transferred to carbon-coated copper grids (200-mesh). No staining was necessary since the inorganic silicate particles have sufficient contrast with the polymer. Differential scanning calorimetry (DSC) was performed in a TA Instruments Q100 under a nitrogen atmosphere operated at $10{ }^{\circ} \mathrm{C} / \mathrm{min}$. Tensile measurements were done on an Instron 5866 frame using injection-molded dog-bone specimens (defined and tested per the ASTM-D638 protocol).

Melt-state rheological measurements were performed on a TA Instruments ARES rheometer with $25 \mathrm{~mm}$ diameter parallel plates and a transducer with torque range of $0.2-2000 \mathrm{~g}_{\mathrm{f}} \mathrm{cm}$. Two types of rheological measurements were performed to investigate the linear and nonlinear response of the nanocomposites.

Dynamic linear frequency sweeps were carried out for three different temperatures using small amplitude oscillatory shear. Sinusoidal strain of the form

$$
\gamma(t)=\gamma_{0} \sin (\omega t)
$$

was applied, where $\gamma_{0}$ is the strain amplitude, $\omega$ the frequency, and $t$ the time. The time-dependent stress was determined as

$$
\sigma(t)=\gamma_{0}\left(G^{\prime} \sin (\omega t)+G^{\prime \prime} \cos (\omega \mathrm{t})\right)
$$

where $G^{\prime}$ and $G^{\prime \prime}$ are the storage and loss moduli respectively.

To examine the nonlinear rheological response of the nanocomposites to steady flow, start-up of steady shear measurements as a function of shear rate $\left(0.02-1 \mathrm{~s}^{-1}\right)$ were performed. Rheological parameters such as shear stress $(\sigma)$ and the first normal stress difference $\left(\mathrm{N}_{12}\right)$ were recorded as a function of time until a steady value is reached. Typically, a rest time between shear rates is allowed for obtaining reproducible results.

\section{Results and Discussion}

3.1. Structural Characterization. From a combination of $\mathrm{X}$-ray diffraction (XRD) and TEM studies, we surmise that the series 1 nanocomposites, based on the PP- $g$-MA/mmt concentrate, exhibit a mixed morphology that contains well-dispersed (exfoliated) montmorillonite platelets, as well as tactoids containing multiple montmorillonite layers. These tactoids of parallel-stacked montmorillonite layers (intercalated morphology) gives rise to a definitive $\mathrm{d}_{001}$ reflection in wide angle XRD (Figure 1). The morphologies were also directly observed by bright field TEM and representative micrographs are given in Figure 2. TEM observations indicate similar composite structures for all three systems, with a slightly better dispersion, more exfoliated single montmorillonite layers and fewer numbers of 


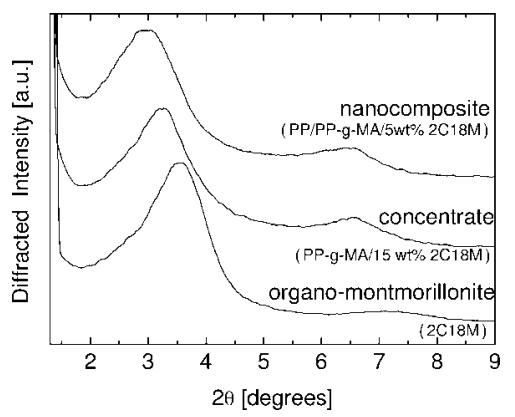

Figure 1. Wide angle X-ray diffraction patterns comparing the organically modified montmorillonite (dimethyl dioctadecyl ammonium exchanged, $2 \mathrm{C} 18 \mathrm{M})$, its $15 \mathrm{wt} \%$ concentrate with maleic anhydrite polypropylene (PP-g-MA/2C18M), and the nanocomposites resulting from dilution of this concentrate in an unfunctionalized PP matrix, All three nanocomposites exhibit similar XRD patterns, here the data for the MNC composite are shown.

larger tactoids, with increasing molecular weight of the PP matrix polymer (cf. Figure 2). For the nanocomposites of series 2 , based on the PP- $t-\mathrm{NH}_{3}{ }^{+} / \mathrm{mmt}$ concentrate, there were no $d_{001}$ reflections observed in the XRD (Figure S-1, Supporting Information), and TEM indicated a disordered (HN191 and HN192 nanocomposites) tactoid morphology, and a mostly exfoliated (HN188 concentrate) morphology, as reported in detail in previous work (Figure S-2, Supporting Information). ${ }^{17}$

SAXS measurements of the series 1 nanocomposites were performed in the melt state of PP to complement the XRD and TEM described above and SAXS measurements previously performed on series $2 .{ }^{17}$ In the melt state of PP, all three series 1 nanocomposites (HNC, MNC, and LNC) exhibit typical power law dependence at low- $q$, i.e., $I(q) \sim q^{-n}$ (Figure 3), over the range $0.06<q<0.4 \mathrm{~nm}^{-1}$. The power law exponents are roughly similar for all the three nanocomposites $(n \sim 2.5 \pm 0.1)$. For well-dispersed fully exfoliated clay dispersions, it is anticipated that for conditions where the individual silicate disk contribution dominates the scattering, the SAXS intensity would scale as $q^{-2}$. For these nanocomposites, the intercalated clay tactoids result in a stronger power-law behavior. In this context, we conclude based on the SAXS data that, consistent with X-ray diffraction measurements, the nanocomposites belonging to series 1 include a significant fraction of intercalated structures. Furthermore, the overall state of dispersion of the layered silicates, to the sensitivity permitted by the SAXS analysis, is roughly the same for all three PP/PP-g-MA/montmorillonite nanocomposites, and no effects of the molecular weight of the PP were manifested in the SAXS data.

3.2. Thermal Characterization. The nanocomposites of series 1 have been further characterized by differential scanning calorimetry (DSC) and compared to the respective PP polymers and blends, i.e., 70/30 by weight blends of PP/PP-g-MA (Table 2 ). The thermal properties of these PP nanocomposites exhibit the typical trends of PP reinforced by organically modified montmorillonite: ${ }^{3,18}$ Specifically, the melting point $\left(T_{\mathrm{m}}\right)$ of the nanocomposite is largely unaffected, and there is a slight change in the crystalline fraction, which is associated with the heterogeneous nucleation of PP crystals by the inorganic. This latter effect is also manifested through an increase in the crystallization temperature $\left(T_{\mathrm{c}}\right)$ upon nonisothermal ramped cooling. The comparison of the DSC data for the nanocomposites against those for the neat polymer and for the PP/PP-g-MA blend, can quantify the competing effects of the montmorillonite, which act largely as heterogeneous nucleating sites, and of the PP- $g$ MA, which tends to decrease the crystallinity of the unfunctionalized polymers. Cross-polarized optical microscopy observations of the nanocomposites, showed the typical decrease in
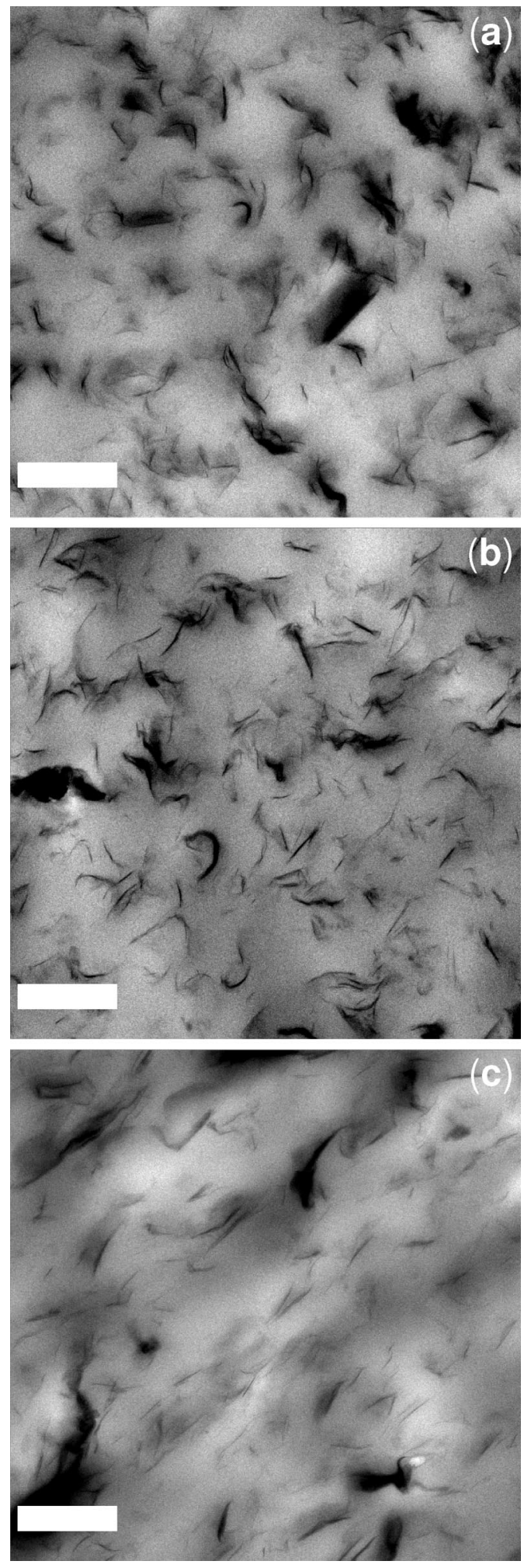

Figure 2. Bright field TEM micrographs of the nanocomposites based on the PP- $g$-MA/2C18 M concentrate: (a) higher molecular weight PP matrix, HNC; (b) medium molecular weight PP matrix, MNC; (c) lower molecular weight PP matrix, LNC. All nanocomposites contain $5 \mathrm{wt}$ $\%$ organo-montmorillonite, and all scale bars are $500 \mathrm{~nm}$.

spherulitic size and the accompanying increase in nucleation density compared to unfilled PP. ${ }^{3,18}$

3.3. Mechanical Properties. The mechanical properties of the nanocomposites, as quantified by tensile stress-strain measurements, are summarized in Table 3. The tensile modulus (Young's modulus) is $10-20 \%$ higher than that corresponding 


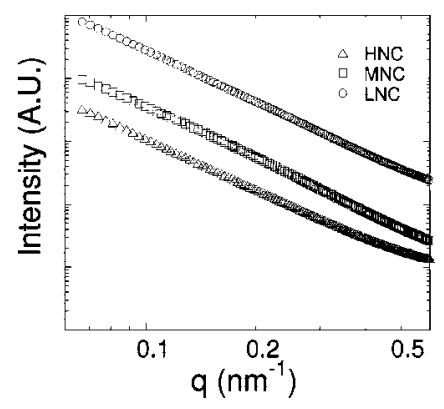

Figure 3. Melt state small-angle X-ray scattering data indicate that all nanocomposites based on the same PP- $g$-MA masterbatch exhibit typical power law dependence $I(q) \sim q^{-n}$ at low $q$, over the range of 0.06 $\mathrm{nm}^{-1}<q<0.4 \mathrm{~nm}^{-1}$. Comparing between the nanocomposites with varying matrix molecular weight (HNC, MNC, LNC), we observe that the SAXS based low $q$ power-laws are similar, indicating roughly similar dispersions of the silicate sheets in these three nanocomposites in the melt.

to the unfilled matrix, and is larger for the lower crystallinity polymers. This improvement in modulus is in concert with the response of $\mathrm{PP} /$ montmorillonite nanocomposites at about $3 \mathrm{wt}$ $\%$ of inorganic ( $5 \mathrm{wt} \%$ of $2 \mathrm{C} 18 \mathrm{M}$ ), when good dispersions are achieved, ${ }^{3}$ and the yield points, i.e., yield stress and strain, remain roughly the same as those of the respective matrix. In contrast to lower modulus polypropylene, ${ }^{3}$ these nanocomposites fail shortly after the yield point ( $c f$. elongations at break in Table 3 ), an embrittlement which is characteristic of nanocomposites of stiff semicrystalline polymers, such as PET, or stiff amorphous polymers, such as polycarbonate.

Although at first glance both the thermal and the mechanical properties of nanocomposites reported here seem to follow the typical behavior of PP/silicate nanocomposites, one final point should be made to elucidate the competition between the "plasticization" effect of the PP- $g$-MA and the reinforcing effect of the montmorillonite layers, beyond any crystal nucleation effects. One way to trace the origins of mechanical reinforcement, i.e., the increase in Young's modulus, for these hybrids is to correlate the tensile modulus to the crystallinity (Figure 4). The correlation between tensile modulus and crystal fraction for the nonreinforced blends is roughly linear, and when extrapolated to the slightly higher crystallinity values corresponding to those of the nanocomposites, it can account for the modulus increase due to the higher crystallinity resulting by the addition of nanoparticles. The excess modulus improvement, i.e., the modulus increase above the extrapolated moduluscrystallinity line defined by the blends, denotes the mechanical reinforcement due to stress transfer from the "softer" matrix to the "stiffer" inorganic particles. The relatively small magnitude of this excess modulus improvement is in concert with the weak interfacial strength of the polyolefin/alkyl-ammonium-modified montmorillonite interface. ${ }^{19,20}$

3.4. Linear Dynamic Oscillatory Response. Master curves of the storage and loss moduli $\left(G^{\prime}\right.$ and $G^{\prime \prime}$, respectively) and the complex viscosity $\left(\eta^{*}\right)$ of the master-batch $(\mathrm{M} / \mathrm{B}$, series 1 with 15 wt \% organoclay) in the melt state are shown in Figure 5a. The low-frequency plateau in $\mathrm{G}^{\prime}$ and the divergence of $\eta^{*}$ in a cross plot of the $\eta^{*}$ and the complex modulus $\left(G^{*}\right)$ (Figure $5 b)$ indicate that the master-batch in the melt state of the polymer exhibits solidlike response to small amplitude oscillatory shear and consistent with a material with a yield stress. This observed solidlike response and associated yield stress of the masterbatch is attributed to the formation of a percolated network structure of intercalated tactoids and exfoliated sheets, as described previously by Ren and co-workers. ${ }^{5}$

In Figure 6, we compare and contrast the melt-state viscoelastic data for the three nanocomposites prepared by diluting the PP- $g$-MA master-batch with neat PP polymers of varied molecular weights (series 1). These three nanocomposites exhibit rheological behavior between that of a liquid and that of a solid at low oscillatory shear frequencies. We observe that at all frequencies the values of $G^{\prime}$ for $\mathrm{HNC}$ are significantly higher than those of MNC and LNC. At high frequency, the observed trend is consistent with the increased molecular weight of the matrix PP. Moreover, the low frequency values of $G^{\prime}$ for HNC are significantly higher than those of MNC and LNC and suggests that there is significant additional reinforcement in the case of the HNC, that would be consistent with better dispersion of the organoclay (i.e., better exfoliation) in HNC compared to $\mathrm{MNC}$ and $\mathrm{LNC}^{21}$

We anticipate that for $5 \mathrm{wt} \%$ of organo-montmorillonite in the nanocomposites the rheology would be strongly dependent on the mesoscale dispersion of the inorganic layers (i.e., percolation of nanoparticle tactoids and of any exfoliated individual sheets) in the polymer matrix. ${ }^{4}$ It has been shown that the mesoscale dispersion and, in particular, the individualization of some of the organoclay sheets in the nanocomposites depend on the processing of the nanocomposites and the molecular weight of the matrix polymers. ${ }^{22}$ Since the PP- $g$ MA in the master-batch and the processing of the different nanocomposites is identical, any differences in dispersion should be primarily due to molecular weight differences between the polymer matrices.

Furthermore, on the basis of the level of maleic anhydride functionalization of the PP- $g$-MA $\left(\phi_{\mathrm{MA}} \sim 0.1 \mathrm{wt} \%\right),{ }^{23}$ we expect that in the melt state the functionalized PP- $g$-MA and the pristine low, medium and high molecular weight PP would be completely miscible and that the results we are observing here are not a result of a phase separated morphology (e.g. melt incompatibility of the functionalized and unfunctionalized PP polymers). Previous studies of blend miscibility of PP and PP$g$-MA using a special staining technique and electron microscopy, ${ }^{24}$ indicated that in order to obtain phase separation from such functional mismatches in polymer structure, the extent of grafting of MA would have to exceed $1 \%$ for the molecular weights used in this study. Thus, for the systems examined here, melt-state phase separation and the consequent morphology can be ruled out as the cause for the observed viscoelastic data in the nanocomposites.

A better rheological measure of the possible better dispersion of the HNC nanocomposite is by comparison of the complex viscosities, especially at low frequencies. In order to quantitatively compare the different nanocomposites, we compare the normalized complex viscosities $\eta_{r}{ }^{*}$ in Figure 7 . The normalization is done by using a measured value of the respective effective zero shear viscosity for the PP- $g$-MA/PP blends (for low, medium and high molecular weight PP, as detailed in Appendix A). Further, in order to compare the data from the different molecular weight samples, we normalize the frequency by the characteristic relaxation time for the matrix blends, here approximated as the reciprocal of the frequency at the crossover of $G^{\prime}$ and $G^{\prime \prime}$. At values of $\omega \tau_{\text {blend }}$ of 0.1 and larger, the normalized viscosities of the nanocomposites were roughly independent of polymer molecular weight, and consistent with the notion that the high frequency viscoelastic response is largely independent of the mesoscale dispersion. On the other hand, at values of $\omega \tau_{\text {blend }}$ significantly smaller than 0.1 , the viscosity response is mainly dictated by the silicate network formed in the nanocomposites and the differences between the nanocomposites are a reflection of the mesoscale dispersion of the silicate layers. Thus, since the HNC nanocomposite exhibits a significant increase in the low frequency viscosity, we conclude that HNC has a better dispersion; i.e., there exists a higher fraction of exfoliated layers in HNC than in MNC and LNC. 
Table 2. Differential Scanning Calorimetry Data for the Systems of Series $\mathbf{1}^{a}$

\begin{tabular}{|c|c|c|c|c|c|c|c|c|}
\hline \multirow[b]{2}{*}{ sample } & \multicolumn{3}{|c|}{ composition (wt \%) } & \multirow[b]{2}{*}{$T_{\mathrm{m}}\left({ }^{\circ} \mathrm{C}\right)$} & \multirow[b]{2}{*}{$T_{\mathrm{c}}\left({ }^{\circ} \mathrm{C}\right)$} & \multirow[b]{2}{*}{$\Delta H_{\mathrm{m}}(\mathrm{J} / \mathrm{g})^{b}$} & \multirow[b]{2}{*}{$\Delta H_{\mathrm{c}}(\mathrm{J} / \mathrm{g})^{b}$} & \multirow[b]{2}{*}{$\Phi_{\mathrm{c}}(\%)$} \\
\hline & $\mathrm{PP}$ & F-PP & $2 \mathrm{C} 18 \mathrm{M}$ & & & & & \\
\hline HPP & 100 & 0 & 0 & 163.9 & 118.7 & 105.3 & 105.3 & 64.6 \\
\hline HBN & 70 & 30 & 0 & 162.7 & 118.7 & 101.7 & 99.1 & 60.8 \\
\hline MPP & 100 & 0 & 0 & 159.0 & 116.2 & 97.5 & 97.7 & 60.0 \\
\hline MBN & 70 & 30 & 0 & 159.0 & 118.1 & 98.2 & 98.0 & 60.1 \\
\hline MNC & 67 & 28 & 5 & 159.6 & 119.4 & 101.8 & 102.2 & 62.7 \\
\hline LNC & 67 & 28 & 5 & 163.1 & 120.4 & 105.2 & 103.3 & 63.3 \\
\hline
\end{tabular}

${ }^{a}$ All DSC scans were done at a $5{ }^{\circ} \mathrm{C} / \mathrm{min}$ ramp rate, under $\mathrm{N}_{2}$, and the results reported are from the second scan. The reported values correspond to the melting point $\left(T_{\mathrm{m}}\right)$, crystallization point $\left(T_{\mathrm{c}}\right)$, enthalpy of melting $\left(\Delta H_{\mathrm{m}}\right)$, enthalpy of crystallization $\left(\Delta H_{\mathrm{c}}\right)$, and crystalline fraction $\left(\Phi_{\mathrm{c}}\right.$, calculated from $\Delta H_{\mathrm{c}}$ and based on $163 \mathrm{~J} / \mathrm{g}$ for the PP crystal). ${ }^{b}$ The enthalpy of melting $\left(\Delta H_{\mathrm{m}}\right)$ and the enthalpy of crystallization $\left(\Delta H_{\mathrm{c}}\right)$ are both reported per gram of polymer, rather than the usual per gram of sample.

Table 3. Tensile Testing Results for the Systems of Series $\mathbf{1}^{a}$

\begin{tabular}{|c|c|c|c|c|c|c|c|c|}
\hline \multirow[b]{2}{*}{ sample } & \multicolumn{3}{|c|}{ composition (wt \%) } & \multirow[b]{2}{*}{$\begin{array}{l}\text { tensile modulus } \\
(\mathrm{MPa})\end{array}$} & \multirow[b]{2}{*}{$\begin{array}{l}\text { yield stress } \\
(\mathrm{MPa})\end{array}$} & \multirow[b]{2}{*}{$\begin{array}{l}\text { elongation at } \\
\text { yield (\%) }\end{array}$} & \multirow[b]{2}{*}{$\begin{array}{l}\text { ultimate strength } \\
\text { (MPa) }\end{array}$} & \multirow[b]{2}{*}{$\begin{array}{c}\text { elongation at } \\
\text { break }(\%)\end{array}$} \\
\hline & PP & F-PP & $2 \mathrm{C} 18 \mathrm{M}$ & & & & & \\
\hline HPP & 100 & 0 & 0 & $1309 \pm 19$ & $35 \pm 0.3$ & $7 \pm 0.2$ & $37 \pm 8$ & $526 \pm 137$ \\
\hline HNC & 67 & 28 & 5 & $1492 \pm 96$ & $33 \pm 0.3$ & $6 \pm 0.2$ & $13 \pm 2$ & $13 \pm 6.2$ \\
\hline MPP & 100 & 0 & 0 & $1254 \pm 80$ & $32 \pm 0.2$ & $8 \pm 0.2$ & $17 \pm 12$ & $356 \pm 156$ \\
\hline MBN & 70 & 30 & 0 & $1160 \pm 24$ & $31 \pm 0.9$ & $7 \pm 0.4$ & $38 \pm 2$ & $629 \pm 52$ \\
\hline LNC & 67 & 28 & 5 & $1443 \pm 30$ & $31 \pm 0.1$ & $5 \pm 0.1$ & $30 \pm 1$ & $5 \pm 0.7$ \\
\hline
\end{tabular}

${ }^{a}$ All experiments were obtained as per ASTM-D638 with a crosshead speed of $25 \mathrm{~mm} / \mathrm{min}$ in an Instron tensile tester.

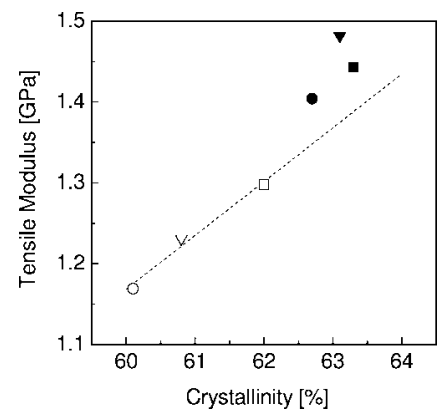

Figure 4. Tensile (Young's) modulus plotted versus crystal fraction for the PP/PP- $g$-MA blends (open symbols) and for the respective PP/ $\mathrm{PP}-g$-MA/2C18 M nanocomposites (filled symbols). All three unfunctionalized polypropylenes are shown (higher $M_{\mathrm{w}}$, triangles; medium $M_{\mathrm{w}}$, circles; and lower $M_{\mathrm{w}}$, squares). The modulus improvement capability of the montmorillonite fillers, beyond their PP crystal nucleation effect, is denoted by the modulus increase in excess of the extrapolated line for modulus vs crystal fraction derived from the blends

In contrast to the reported viscoelastic behavior of the PP$g$-MA based nanocomposites of series 1 (described above), the linear viscoelastic melt-rheological data for the $\mathrm{NH}_{3}{ }^{+}$terminated PP based nanocomposites (series 2) display liquid-like response in the low frequency region for all three nanocomposites (Figure 8). It has been proposed that $\mathrm{NH}_{3}{ }^{+}$terminated PP interact with the layered silicates directly through ionic interaction, resulting in end-tethering of the polymers and an exfoliated structure of the silicates ${ }^{17}$ (Scheme 1a). However, this single interaction site of the polymer with the silicate surface is evidently insufficient for the formation/stabilization of a long-lived silicate network, mediated by polymer chains that bridge across nanoparticles (Scheme 1a). Therefore, these nanocomposites, based on endtethered polymers attached to montmorillonite layers, exhibit weak interactions between the polymer chains and the silicates and results in the liquid-like terminal behavior. Clearly, the absence of a long-lived three-dimensional silicate network and
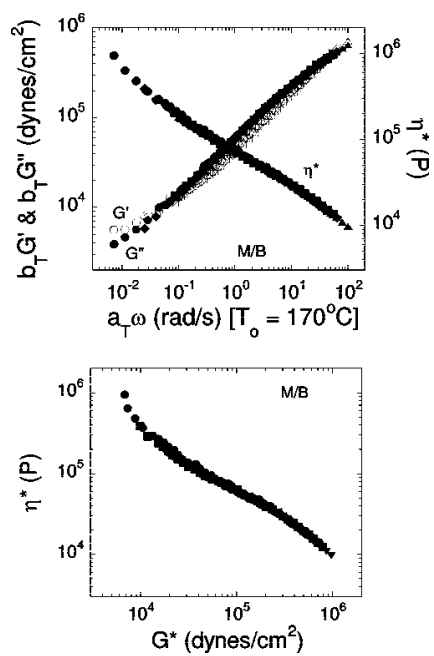

Figure 5. Melt state dynamic linear viscoelasticity of the maleic anhydride functionalized master-batch nanocomposite, superposed at $170^{\circ} \mathrm{C}$ to create a rheological master-curve. The presence of solid like behavior and a yield stress is attributed to the formation of a percolated silicate network that is presumably stabilized by the bridging interactions caused by the multisticker MA grafted polymer chains.

the inherent lack of attractive interactions between PP and the layered silicate, result in the dissipation of the stress via relaxations of the polymer chains, which is manifested macroscopically in a liquid-like behavior.

In contrast, the MA-functionalized PP chains are able to form at most two contacts per chain with the silicate layers and further because of physical entanglements of such doubly tethered polymers leads to effective bridging interactions between the nanoparticles $^{25-27}$ (Scheme 1b), and to the development of an extended and possibly hierarchical superstructure which is manifested macroscopically in the solid-like behavior. Thus, even though the individual MA group interactions with the silicate are weaker than the corresponding ammonium cation 


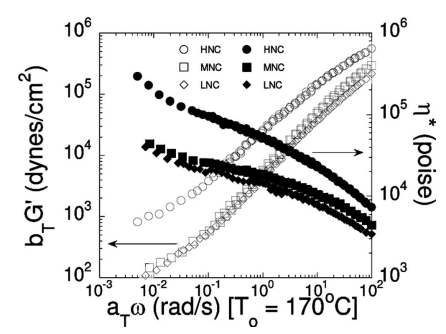

Figure 6. Melt state dynamic linear viscoelasticity of the maleic anhydride functionalized nanocomposites diluted from the master-batch superposed at $170{ }^{\circ} \mathrm{C}$. The high molecular weight nanocomposite exhibits stronger solid like behavior presumably due to better silicate dispersion.

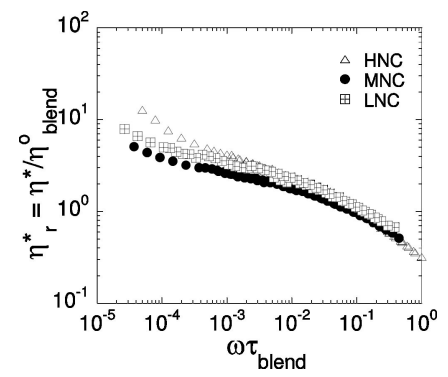

Figure 7. Normalized complex viscosity as a function of dimensionless time scale. The viscosities are normalized by the zero shear viscosity of the blend of the modified and unmodified polymers and the time scale is normalized by the longest relaxation time of the blend. The high frequency response is largely independent of the mesoscale dispersion. On the other hand, at small values of $\omega \tau_{\text {blend }}$, the viscosity response is mainly dictated by the silicate network formed in the nanocomposites.

interactions with the silicate layers, ${ }^{28}$ the MA-treated PP can promote the formation of a filler network structure -and consequently result in a solid-like rheological response- because of their ability to effectively bridge silicate sheets. Similar filler networks, and the same qualitative rheological trend, have also been observed in montmorillonite filled nanocomposites of polyolefin random copolymers, ${ }^{8}$ namely polyethylene-randomvinyl acetate (EVA); however, for these EVA systems, a much higher polar comonomer fraction is necessary ( $28 \mathrm{wt} \%$ of VA was used in that study ${ }^{8}$ ) to achieve a comparable solidlike response with the one observed here. In addition, the polymernanoparticle interfacial adhesion in nanocomposites based on PE- $r$-VA/mmt master-batches diluted by PE are substantially weaker than the ones of the respective PE- $g$-MA/mmt masterbatch based nanocomposites. ${ }^{20}$ These trends for the EVA based nanocomposites further attest to the effectiveness of the MAtreated polyolefins in stabilizing layered-silicate filler networks, in support of the conclusions from this present study.

3.5. Steady Shear Viscoelasticity. Steady shear, in start-up mode, for the three nanocomposites of series 1 (HNC, MNC, and LNC) were also performed, in order to understand the response to shear flow of the mesoscale structure of the layered silicates dispersed in the polymer. These three nanocomposites behaved similarly and, for the sake of clarity, we will focus on the time-dependent evolution of the shear stress for the low molecular weight PP nanocomposite (LNC). Figure 9 displays the transient shear stress response of LNC in the melt state as a function of time over a range of applied shear rates. After a clear overshoot at short times, the stress rapidly equilibrates to a steady plateau with no observation of significant oscillations in the shear stress (or the normal stress). The observed overshoot in the transient shear stress was somewhat surprising considering the molecular weight of the polymers used in this study. To verify the origins of the stress overshoot in the nanocomposites,
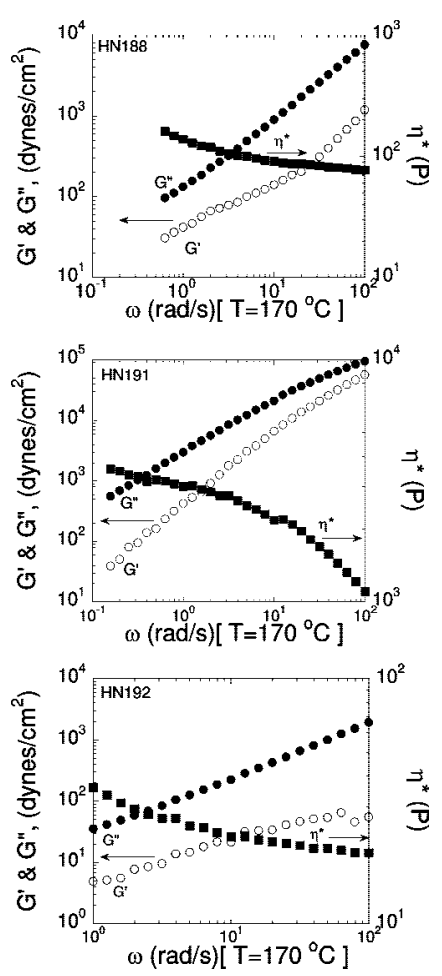

Figure 8. Melt state dynamic linear viscoelasticity of the ammonium functionalized nanocomposites at $170{ }^{\circ} \mathrm{C}$. The master-batch and the ones diluted from the master-batch all exhibit liquid like behavior despite being well dispersed. The absence of the formation of a percolated silicate network results in weak interaction between the polymer chains and the silicates.

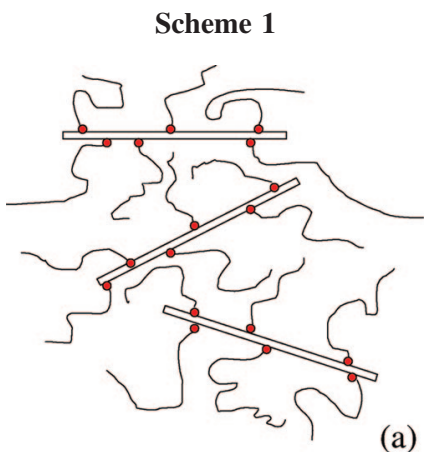

(a)

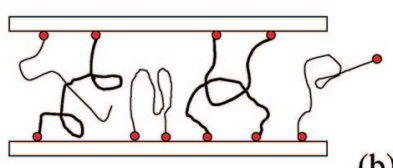

(b)

we performed the same start-up mode steady shear experiment on the unfilled polymers, and these experiments showed an absence of stress overshoot in those matrices up to a shear rate of $10 \mathrm{~s}^{-1}$. We thus suggest that the overshoot in the transient shear stress for the nanocomposites is related to the presence of dispersed silicate sheets or tactoids and the resistance to deformation offered by the mesoscale superstructure. ${ }^{27,29}$ Specifically, the magnitude of the transient shear stress overshoot exhibits a power law dependence on shear rate, with $\sigma_{\max } \sim$ $(\dot{\gamma})^{0.5}$ (Figure 9) and the ratio $\left(\left(\sigma_{\max } / \sigma_{\infty}\right)-1\right)$, where $\sigma_{\infty}$ is the steady state shear stress (Figure 10), is largely independent of the strain rate. This latter point indicates that the transient stress overshoot, much like the steady shear value of the stress, is largely a reflection of the mesoscale structure of the nanocomposites and its orientation due to the application of shear. ${ }^{30-32}$ 


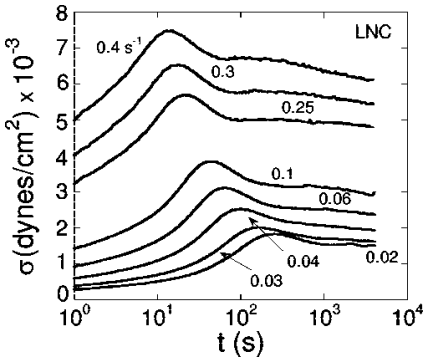

Figure 9. Transient shear stress in startup of steady shear is monitored as a function of time. Surprisingly, a transient shear stress overshoot is observed across all shear rates. This overshoot is not seen in the homopolymers under similar experimental conditions. It is therefore a result of orientation of the silicates and its nanocomposites in response to steady shear.

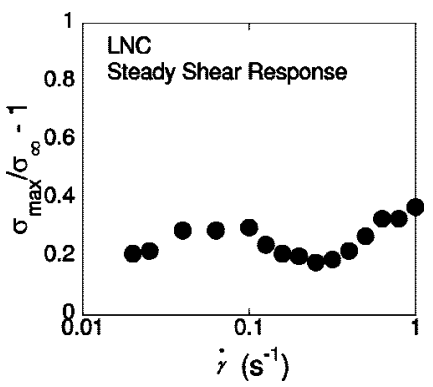

Figure 10. $\left.\left(\sigma_{\max } / \sigma_{\infty}\right)-1\right)$ as a function of shear rates, where $\sigma_{\infty}$ is the steady state shear stress. This ratio is largely independent of stress rates, suggesting that stress shear overshoot is mainly a reflection of the structure and the orientation of the silicate sheets and the tactoids of silicate sheets due to the application of shear.

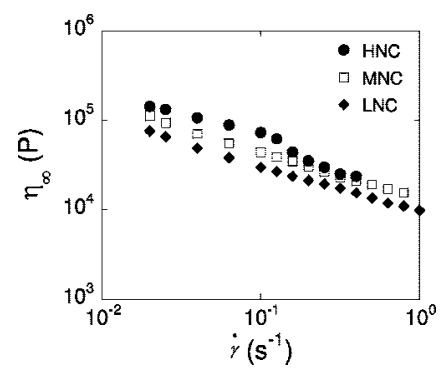

Figure 11. Steady shear viscosity as a function of shear rates for the three nanocomposites prepared using the PP- $g$-MA. The data for all three samples indicates that $\eta \sim(\dot{\gamma})^{-0.5 \pm 0.1}$ and somewhat weaker than the frequency dependence of the complex viscosity for the same systems.

The ratio of $\left(\sigma_{\infty} / \dot{\gamma}\right)$ is the steady shear viscosity, $\eta(\dot{\gamma})$, and its dependence on $\dot{\gamma}$ is shown in Figure 11. The steady shear viscosity clearly exhibits a non-Newtonian behavior and follows a power-law scaling of $\eta_{\infty} \sim(\dot{\gamma})^{-0.5 \pm 0.1}$, which is somewhat weaker than the low frequency power-law dependence of $\eta^{*}$ obtained with small strain oscillatory shear, i.e., at rheological conditions that are not expected to disturb the quiescent mesoscale structure. This suggests that the application of steady shear results in some orientation of the silicate sheets and tactoids in response to the applied shear. ${ }^{32}$ Furthermore, this orientation is shear rate dependent and is manifested by the rather strong dependence of $\sigma_{\max }$ and $\eta$ on $\dot{\gamma}$. In this context, an analytic rheological model derived by Malamataris and Papanastasiou $^{33}$ qualitatively captures the observed dependence: specifically, this model, developed to describe the shear deformation of nondilute, slender-fiber suspensions in Newtonian media, is valid under the assumption of random initial fiber orientation. It predicts an overshoot in the stress which is only a function of the total strain $(\gamma=\dot{\gamma} \times t)$, and furthermore it predicts that the viscosity of the suspension approaches that of

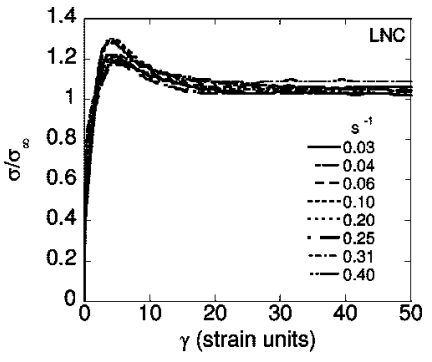

Figure 12. Magnitude of the transient stress overshoot normalized by steady shear stress values as a function of strain units $(\dot{\gamma} \times t)$. It is clear that the shear stress overshoot occurs at roughly the same strain for the shear rates examined.

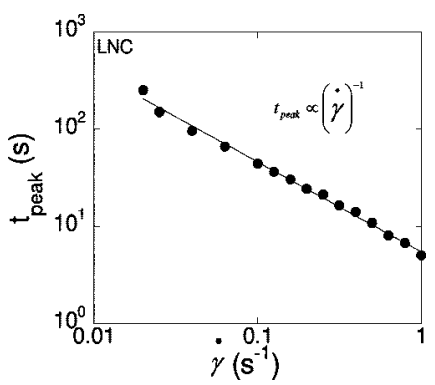

Figure 13. Time at which the shear stress overshoot is observed as a function of shear rates. The unique strain scaling of stress is characteristic of materials having non-Brownian behavior.

the matrix due to the orientation of the nanoparticles. These predictions are consistent with the strain dependence of the transient shear overshoot observed in this study: the presence of the stress overshoot, which we have previously demonstrated as a result of the mesoscale structure of the layered silicates, is consistent with this model. Further, the transient shear stress, and in particular the location of the stress overshoot, scales with the total applied strain $(\dot{\gamma} \times t)$ (Figure 12); also, the time after inception of flow at which the primary peak in the stress response was observed was plotted as a function of $\dot{\gamma}$ and is consistent with the scaling $t_{\text {overshoot }} \sim(\dot{\gamma})^{-1}$ (Figure 13). ${ }^{34}$ These results, similar to those from previous studies ${ }^{31,34}$ indicate that the stress overshoot exhibits a shear strain scaling. Such behavior is typically observed in materials that possess no characteristic time scale, such as textured liquid crystalline polymers, ${ }^{35}$ nonBrownian suspensions of rods and disks, ${ }^{36}$ and some layered silicate nanocomposites. ${ }^{30,37,38}$ This is not surprising, since for the viscosity of the polymers employed in this study and the lateral dimension of the layered silicates, the rotational Peclet number is much smaller than one. ${ }^{36}$ Thus, it is inferred that Brownian motion of these layered silicates is rather limited and that the particle-particle interaction within the nanocomposites would ultimately dominate the nature of the shear flow. ${ }^{5,21,37-39}$

It has been reported previously that such layered silicate based nanocomposites demonstrate relatively small effects on the first normal stress difference $\left(N_{12}\right)$ when compared at equivalent values of shear stress. ${ }^{38}$ The relationship between $N_{12}$ and $\sigma_{\infty}$ obtained at long times is shown in Figure 14 for each nanocomposite. At large values of shear stress, the relationship between $N_{12}$ and $\sigma_{\infty}$ exhibit only minor differences between the nanocomposites with different molecular weight PP, and show $N_{12}$ scaling as $\sigma_{\infty}{ }^{-0.5 \pm 0.01}$. This result can be compared with the empirical form suggested by Mall-Gleissle: ${ }^{40}$

$$
N_{12}(\varphi, \sigma)=A(\varphi) \sigma_{12}^{n^{\prime}}
$$

Here $A$ is a $\phi$ (i.e., volume fraction) dependent quantity representing the nanoparticle contribution to $N_{12}$ and $n^{\prime}$ denotes a volume fraction independent exponent describing the rheology 


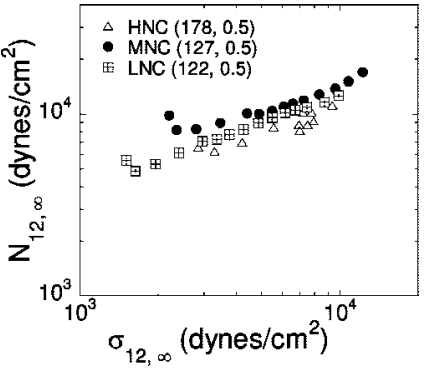

Figure 14. Steady normal stress as a function of steady shear stress. On the basis of the values of $A$ and $n$, substantial shear induced alignment of layered silicate occurs at high shear rates. In addition, the weaker dependence of $N_{12}$ on $\sigma_{\infty}$ implies that the nanocomposites reinforced by the layered silicate network may reduce the magnitude of $N_{12}$ and effectively suppress die-swelling during processing.

of the polymer. In Figure 14 , the parameters $(A, n)$ for a fit of eq 4 to our data are displayed. Interestingly, $n \approx 0.5$ for all the nanocomposites studied, whereas for monodisperse homopolymers $n$ is anticipated to be roughly two. ${ }^{41}$ This weaker dependence of $N_{12}$ on $\sigma_{\infty}$ implies that the nanocomposites reinforced by the layered silicate network may reduce the magnitude of $N_{12}$ and effectively suppress die-swelling during processing. ${ }^{4,29}$ This observation is analogous to that in the work of Kharchenko and co-workers, ${ }^{42}$ where they demonstrated the presence of a negative normal stress difference in nanotubefilled polypropylene nanocomposites and attributed it to the response of the mesoscale nanoparticle network to stretch in the flow direction while compressing along the flow gradient direction. Therefore, it is conceivable that the layered silicate network mediated by bridging interaction by polymer chains also exhibits such flow induced behavior.

\section{Concluding Remarks}

Using linear and nonlinear viscoelastic rheological measurements, we have contrasted the rheological properties of layered silicate nanocomposites prepared using a maleic anhydride grafted polypropylene (with a maximum of two stickers) masterbatch with those prepared using a single-end ammonium functionalized polypropylene (single sticker) master-batch. The single ammonium terminated PP based nanocomposites exhibit liquid-like rheological behavior, in spite of the highly dispersed state of the silicate sheets and the expectation of a filler-network dominated response. We suggest that the presence of a singleend functionalized PP, while providing sufficient thermodynamic driving force for the dispersion of the silicate layered-fillers, is unable to sustain a polymer mediated filler network structure, which is evidently weakened by the fast relaxation of the topological entanglements that might otherwise allow for bridging interactions in these nanocomposites. In contrast, the nanocomposites based on maleic anhydride functionalized PP exhibit rheological behavior that is solidlike and consistent with the notion of a percolative filler network structure. While the achieved dispersion of the layered silicates in these systems was not as good as the dispersions obtained by the single endfunctionalized ammonium PP, the presence of more-than-one interaction sites per polymer chain allows for long-lived bridging interactions between silicate sheets or their tactoids and, therefore, a percolated superstructure. These results, along with the steady-shear response of the quiescently percolated structures, indicate that the silicate sheets act as non-Brownian objects; moreover, the inclusion of these platelets leads to minimal changes in the first normal stress difference, indicating a distinctive role of polymer/nanofiller interactions and bridging on the reinforcement mechanism in such nanocomposites. We also believe that these results, obtained from a systematic series
Table 4. Determination of the Viscosity of MA-PP Using the Mixing Rule Described in Equation A-1

\begin{tabular}{ccc}
\hline $\begin{array}{c}\eta_{\text {blend }}, \mathrm{Pa} \\
(\text { measured })\end{array}$ & $\begin{array}{c}\eta_{a, \mathrm{PP}}, \mathrm{Pa} \\
(\text { measured })\end{array}$ & $\begin{array}{c}\eta_{b, \mathrm{MA}-\mathrm{PP}}, \mathrm{Pa} \\
\text { (calculated) }\end{array}$ \\
\hline $2.90 \times 10^{4}$ & $7.00 \times 10^{4}$ & 332 \\
$1.97 \times 10^{3}$ & $3.00 \times 10^{3}$ & 341 \\
$5.50 \times 10^{2}$ & $5.60 \times 10^{2}$ & 321
\end{tabular}

of comparable polymer matrices, clearly establishes that the principle mechanism responsible for the changes in viscoelastic behavior in such nanocomposites is through a polymer mediated filler network (as previously reported extensively on a wide range of systems $4,5,7,9,11,14,21,25,29,37,38,43$ ) and not necessarily through direct particle-particle interactions, although such jamming might become the dominant mechanism at significantly higher loadings of nanoparticles.

Acknowledgment. We gratefully acknowledge T. C. Chung, J. Y. Dong and Z. M. Wang for synthesizing the PP- $t-\mathrm{NH}_{2}$ polymers and Sumitomo Chemical, Japan, for generous donations of several PP grades. R.K. and L.X. acknowledge the financial support from Total Petrochemicals and the National Science Foundation (NSF BES-0609387). We thank the user program at the National Synchrotron Light Source at Brookhaven National Laboratory for the X-ray time on beamline X27C. The work at Penn State was supported by Sumitomo Chemical, Japan, by the ONR (Award No. N000140510614), and through the National Science Foundation (NSF DMR-0602877).

\section{Appendix A}

The matrix for the HNC, MNC and LNC is a 70/30 blend of unmodified PP with varied molecular weight and a single MA-grafted PP polymer. The viscoelastic response of each of the matrices is slightly different due to the molecular weight difference of the unmodified PP employed. The zero-shear viscosity of the three unmodified PPs and their blends with the MA-treated PP (HPP, MPP, LPP, and HBN, MBN, LBN respectively) were measured using standard rheological testing methods and the values reported in the first two columns in Table 4; the values for the blends are used in the construction of the reduced viscosity plots described in Figure 5.

The data obtained are shown to be in excellent agreement with the predictions of simple mixing rules for entangled polymer systems. We applied the mixing rule of Friedman and Porter, ${ }^{44}$ and Struglinski and Graessley ${ }^{45}$ to determine the dependence of the zero-shear viscosity of the blends:

$$
\eta^{1 / c}=w_{a} \eta_{a}^{1 / c}+w_{b} \eta_{b}^{1 / c}
$$

where $c$ is the scaling exponent of $\eta$ on $\mathrm{M}$ (i.e., $\eta \propto \mathrm{M}^{c}$ and typically $c=3.4), \eta$ is the zero-shear viscosity of the blends, $\eta_{a}$ is the zeroshear viscosity of the unmodified PP, $\eta_{b}$ is the zero-shear viscosity of the MA-grafted PP, and $w_{i}$ is the corresponding weight fraction of component $i$ in the blend. On the basis of eq 1, we can calculate the value of $\eta_{b}$ from the measured values of the viscosity of the blend and the corresponding unfunctionalized PP (Table 4) and these indicate a consistent value of $\eta_{b}$ of $330 \pm 10 \mathrm{P}$.

Supporting Information Available: Text discussing the synthesis and characterization of the nanocomposites belonging to series 2 , a scheme showing the synthesis, and figures showing wide angle X-ray diffraction patterns and bright field TEM micrographs. This material is available free of charge via the Internet at http:// pubs.acs.org.

\section{References and Notes}

(1) Krishnamoorti, R. MRS Bull. 2007, 32, 341-347.

(2) Douglas, L. Hunter; Karl, W. Kamena; Paul, D. R. MRS Bull. 2007, $32,323-327$. 
(3) Manias, E.; Touny, A.; Wu, L.; Strawhecker, B.; Lu, B.; Chung, T. C. Chem. Mater. 2001, 13, 3516-3523. (a) Yuan, Q.; Misra, R. D. K. Polymer 2006, 47, 4421-4433. (b) Chrissopoulou, K.; Altintzi, I.; Andrianaki, I.; Shemesh, R.; Retsos, H.; Giannelis, E. P.; Anastasiadis, S. H. J. Polymer Sci., Part B: Polym. Phys. 2008, 46, 2683-2695.

(4) Krishnamoorti, R.; Yurekli, K. Curr. Opin. Colloid Interface Sci. 2001, 6, 464-470.

(5) Ren, J. X.; Silva, A. S.; Krishnamoorti, R. Macromolecules 2000, 33, 3739-3746.

(6) Schmidt, D.; Shah, D.; Giannelis, E. P. Curr. Opin. Solid State Mater. Sci. 2002, 6, 205-212.

(7) Salaniwal, S.; Kumar, S. K.; Douglas, J. F. Phys. Rev. Lett. 2002, 89, 258301.

(8) Gupta, R. K.; Pasanovic-Zujo, V.; Bhattacharya, S. N. J. NonNewtonian Fluid Mech. 2005, 128, 116-125.

(9) Krishnamoorti, R.; Giannelis, E. P. Macromolecules 1997, 30, $4097-$ 4102.

(10) Krishnamoorti, R.; Giannelis, E. P. Langmuir 2001, 17, 1448-1452.

(11) Giannelis, E. P.; Krishnamoorit, R.; Manias, E. Adv. Polym. Sci. 1999, 138, 107-147.

(12) Usuki, A.; Hasegawa, N.; Kato, M., Polymer-clay nanocomposites. In Inorganic Polymeric Nanocomposites and Membranes; 2005, 179, 135-195; Usuki, A.; Kojima, Y.; Kawasumi, M.; Okada, A.; Fukushima, Y.; Kurauchi, T.; Kamigaito, O. J. Mater. Res. 1993, 8, 1179 1184.

(13) Strawhecker, K. E.; Manias, E. Chem. Mater. 2000, 12, 2943-2949. Strawhecker, K.; Manias, E. Macromolecules 2001, 34, 8475-8482.

(14) Pryamitsyn, V.; Ganesan, V. J. Chem. Phys. 2005, 122, 104906.

(15) Liff, S. M.; Kumar, N.; McKinley, G. H. Nat. Mater. 2007, 6, 76-83. (a) Manias, E. Nat. Mater. 2007, 6, 9-11.

(16) Dong, J. Y.; Wang, Z. M.; Han, H.; Chung, T. C. Macromolecules 2002, 35, 9352.

(17) Wang, Z. M.; Nakajima, H.; Manias, E.; Chung, T. C. Macromolecules 2003, 36, 8919-8922.

(18) Manias, E.; Polizos, G.; Nakajima, H.; Heidecker, M. J. Fundamentals of Polymer nanocomposite technology; J. Wiley and Sons: Hoboken, NJ, 2007; pp 31-66.

(19) Zhang, J.; Manias, E.; Wilkie, C. A. J. Nanosci. Nanotechnol. 2008, $8,1597-1615$.

(20) Zhang, J.; Manias, E.; Polizos, G.; Huh, J. Y.; Ophir, A.; Songtipya, P.; Jimenez-Gasco, M. M. J. Adhes. Sci. Technol., 2009, 23, 709-737. Manias, E.; Zhang, J.; Huh, J. Y.; Manokruang, K.; Songtipya, P.; Jimenez-Gasco, M. M. Macromol. Rapid Commun. 2009, 30, 17-23.

(21) Mitchell, C. A.; Krishnamoorti, R. J. Polym. Sci.: Part B, Polym. Phys. 2002, 40, 1434-1443.

(22) Fornes, T. D.; Paul, D. R. Polymer 2003, 44, 4993-5013. Kim, K. N.; Kim, H.; Lee, J. W. Polym. Eng. Sci. 2001, 41, 1963-1969. Yoon, P. J.; Hunter, D. L.; Paul, D. R. Polymer 2003, 44, 5323-5339.

(23) Duvall, J.; Sellitti, C.; Myers, C.; Hiltner, A.; Baer, E. J. Appl. Polym Sci. 1994, 52, 207-216. Duvall, J.; Sellitti, C.; Myers, C.; Hiltner, A.; Baer, E. J. Appl. Polym. Sci. 1994, 52, 915-206.
(24) Gonzalez-Montiel, A.; Keskkula, H.; Paul, D. R. J. Polym. Sci., Part B: Polym. Phys. 1995, 33, 1751. Huang, J. J.; Keskkula, H.; Paul, D. R. Polymer 2006, 47, 624.

(25) Joshi, Y. M. Langmuir 2005, 21, 9013-9016.

(26) Goodman, D.; Kizhakkedathu, J.; Brooks, D. E. Langmuir 2004, 20, 2333-2340.

(27) Chatterjee, T.; Jackson, A. J.; Krishnamoorti, R. J. Am. Chem. Soc. 2008, 130, 6934-6935. (a) Chatterjee, T.; Krishnamoorti, R. Phys. Rev. E 2007, 75, 050403.

(28) Dumont, M.-J.; Reyna-Valencia, A.; Emond, J.-P.; Bousmina, M. J. Appl. Polym. Sci. 2006, 103, 618-625.

(29) Krishnamoorti, R.; Ren, J. X.; Silva, A. S. J. Chem. Phys. 2001, 114, 4968-4973.

(30) Solomon, M. J.; Almusallam, A. S.; Seefeldt, K. F.; Somwangthanaroj, A.; Varadan, P. Macromolecules 2001, 34, 1864-1872.

(31) Treece, M. A.; Oberhauser, J. P. Macromolecules 2007, 40, 571-582.

(32) Chatterjee, T.; Krishnamoorti, R. Macromolecules 2008, 41, 53335338.

(33) Malamtaris, N.; Papanastasiou, T. C. J. Rheol. 1991, 35, 449-464.

(34) Solomon, M. J. Macromolecules 2001, 34, 1864-1872.

(35) Beekmans, F.; Gotsis, A. D.; Norder, B. J. Rheol. 1996, 40, 947-966.

(36) Larson, R. G., The Structure and Rheology of Complex Fluids; Oxford University Press: Oxford, U.K., 1999.

(37) Ren, J. X.; Casanueva, B. F.; Mitchell, C. A.; Krishnamoorti, R. Macromolecules 2003, 36, 4188-4194.

(38) Ren, J. X.; Krishnamoorti, R. Macromolecules 2003, 36, 4443-4451.

(39) Goel, V.; Chartterjee, T.; Bombalski, L.; Yurekli, K.; Matyjaszewski, K.; Krishnamoorti, R. J. Polym. Sci., Part B: Polym. Phys. 2006, 44, 2014-2023.

(40) Mall-Gleissle, S. E.; Gleissle, W.; McKinley, G. H.; Buggisch, H. Rheol. Acta 2002, 41, 61-76.

(41) Han, C. D.; Jhon, M. S. J. Appl. Polym. Sci. 1986, 32, 3809-3840.

(42) Kharchenko, S. A.; Douglas, J. F.; Obrzut, J.; Grulke, E. A.; Migler, K. B. Nat. Mater. 2004, 3, 564-568.

(43) Mitchell, C. A.; Krishnamoorti, R. Macromolecules 2007, 40, 15381545. Pryamitsyn, V.; Ganesan, V. J. Rheol. 2006, 50, 655-683. Schmidt, G.; Nakatani, A. I.; Butler, P. D.; Han, C. C. Macromolecules 2002, 35, 4725-4732. Schmidt, G.; Nakatani, A. I.; Butler, P. D.; Karim, A.; Han, C. C. Macromolecules 2000, 33, 7219-7222. Yurekli, K.; Krishnamoorit, R.; Tse, M. F.; Mcelrath, K. O.; Tsou, A. H.; Wang, H.-C. J. Polym. Sci., Part B: Polym. Phys. 2001, 39, 256-275. Xu, L.; Reeder, S.; Thopasridharan, M.; Ren, J.; Shipp, D. A.; Krishnamoorti, R. Nanotechnology 2005, 16, S514-S521. Meincle, O.; Hoffmann, B.; Dietrich, C.; Friedrich, C. Macromol. Chem. Phys. 2003, 204, 823-830. Mitchell, C. A.; Bahr, J. L.; Arepalli, S.; Tour, J. M.; Krishnamoorti, R. Macromolecules 2002, 35, 8825-8830.

(44) Friedman, E. M.; Porter, R. S. Trans. Soc. Rheol. 1975, 19, 493-508.

(45) Graessley, W. W.; Struglinski, M. J. Macromolecules 1986, 19, 17541760. (a) Struglinski, M. J.; Graessley, W. W.; Fetters, L. J. Macromolecules 1988, 21, 783-789.

MA9002853 